\title{
Threshold-Sensitive Energy Efficient Network and Low Energy Adaptive Clustering Hierarchy Protocols' Performance Appraisal in Wireless Sensor Networks
}

\author{
Asim Saif ${ }^{1}$, Jesse S. Jin ${ }^{2}$, Muhammad Qasim Yasin ${ }^{3}$, Ahmed Mateen ${ }^{4}$ Salman Afsar ${ }^{5}$ and Abdul Rehman \\ ${ }^{1,2}$ College of Intelligence and Computing, Tianjin University, Tianjin, China. \\ ${ }^{3}$ College of Intelligence and Computing, Tianjin University, Tianjin Key Laboratory of Cognitive Computing and \\ Application, Tianjin, China \\ ${ }^{4,5}$ Computer Science Department, University of Agriculture, Faisalabad, Pakistan. \\ ahmedbuttar@uaf.edu.pk \\ ${ }^{6}$ College of Intelligence and Computing, Tianjin University,Tianjin Key Laboratory of Advance Networking, \\ Tianjin, China
}

\begin{abstract}
Due to the inability of being frequently charged the sensor nodes in the WSN in non-accessible milieu is the main concern of enhancing battery lifetime. And for its WSN protocols play a pivotal role. Effectiveness of wireless sensor networks (WSN) varies on routing protocols. The WSN comprise of an enormous number of little nodes that procedure, store or transfer information inside constrained range on account of their little size nodes. Installed sensors are broadly utilized in an alternate sort of uses, for example, tracking or health checking. Information sent with a particular measure of normal start to finish delay, throughput, and jitter in these systems. Multiple protocols are used to overcome that con. Routing helps the (WSN) to pave the way towards better usage of limited powered batteries. The humongous evolvement in the sensor network had been made due to enhancement in the demand of niche solutions for the limited and scarce energy support in WSN. Hierarchical routing is one of the paramount routing techniques which dells with the alike dissemination of burden in network, foremost to the lifetime enchantment of the network. Performance of Threshold-sensitive Energy Efficient Network (TEEN) protocol and Low Energy Adaptive Clustering Hierarchy (LEACH) protocols will be paralleled. This study will be examinee a fore mentioned protocols on the basic of entire energy consumed and sensors' lifespan and make available the appraisal of TEEN and LEACH with imitation established results. Mainly the futures like energy, node alive, nodes data and period will compare. CBR and Video traffic will be used in WSN to compare these features. The aim of aforementioned routing protocols is to achieve a
\end{abstract}

more lengthy actuality of nodes. Another aim of this research is to create the communication amongst nodes and the Base Station (BS) cost-effectively. For simulation, a tool NS-II will be used. The conclusions of this research will be worthwhile for various purposes either non-domestic or domestic.

Key words : LEACH protocol, TEEN, WSN, Base Station, Sensors

\section{INTRODUCTION}

Entrepreneurship is the process of building a new value by WSN are becoming common in the infrastructures segment in the 1970s. These WSN are alienated into two kinds: less infrastructure and networks of infrastructure. Mobil capable hubs can relocate in framework arranges yet base classes remain fixed, when hubs go outdoor the scope of that BS, they enter the assortment of new BS throughout their correspondence. While less system hubs can move during cooperation inside specially appointed which are likewise perceived as offices, they don't have fixed base stations. Portable hubs can set up the directing with one another in specially appointed systems to develop their own' on the fly ' organize. Where every hub, because of the rapidly evolving topology, can go about as both host and switch [1].

WSNs acts as the primary role here in delivering information from the outbound fields to the centralized location. This is how WSNs are implemented in distinct places for distinct purposes. The fundamental elements are quantity of wireless nodes together with base destination (BS). These tiny nodes with devices are cheap and have restricted power and storage ability owing to their tiny design. These nodes can directly send the information to the location depending on the network size [2]. These wireless sensors measure encompassing conditions in the setting in the wake of 
handling these estimations that can help get to the exact state of the fields. To refuge the huge system inclusion, it's imperative to utilize the quantity of hubs that utilization sensors, since radio scope of these little sensors is restricted so it can expand the system size.

These systems can be utilized for some sorts of utilization wellbeing perceptions, military site natural observing and occasion discovery through quick advancement and developing development of WSNs. There is next to no or no foundation in wireless sensor systems [3]. Every hub detect, process information and speak to others. Contingent upon utilization the wireless SN is composed in an irregular or authoritative way. In unsafe territories, all these sensor hubs are kept haphazardly anyway they certainly set in zones that are sheltered. The steering strategies utilized in these sorts of systems contrast from the habitually utilized directing procedures as they utilize an unsure topology. On the off chance that we just utilize one association for directing between the two hubs for information transmission, yet in the event that the subsequent data flops because of any issue, it might lose and correspondence will stop as it doesn't achieve its goal. We use multi-way steering system for the sensor system to diminish or dispose of this issue. On top of it, a system association comes up short, the data can be moved from another connection because of the different ways between hubs [4]. Proactive steering conventions are the directing conventions that hold the directing to any area whatsoever occasions. Yet, when on-demand routing way conventions are uncovered and protected exactly when these courses are required, half breed directing conventions that can work like these conventions [5]. The hubs can openly join or leave a system in these sorts of systems on the grounds that these systems have no foundation. Wireless associations are utilized to connect the hubs to one another. Every hub can act as a switch to send data to its adjoining hubs, which is the manner by which we call these systems less systems as offices. Impromptu systems have the ability to deal with any progressions because of changes in the topology of the system and furthermore have no brought together administration. On the off chance that a hub is down or left its system, it breaks the association between different hubs that are connected through this hub, at that point these influence hubs can demand the new switches in the system to make crisp associations in such system types. As appeared in Fig. 1, it is sorted into three systems.

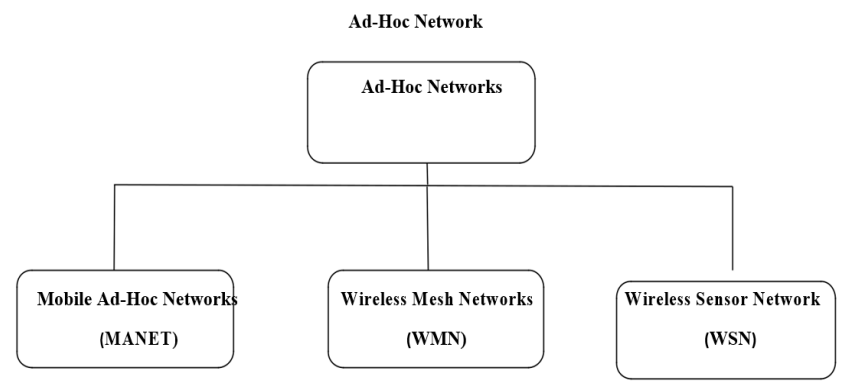

Figure 1: Wireless Ad-Hoc Network Types

\subsection{MANET and WSN}

A few basic distinctions between MANET and WSNs are discussed here. In MANET, there are two terms which can be utilized but not valuable on account of WSNs, where some other steering strategies, for example, one--to--many, many--to--many, can be considered. Disappointment danger of directing are low on account of MANETs however risk is extremely high on account of WSNs [6].

- WSN is strongest and restricted cash than MANETs.

\subsection{Features of WSN}

The wireless system at present includes wireless LAN, impromptu system, Bluetooth organize, portable system, etc. Like the Ad hoc system, this sensor system has numerous attributes like versatility and confined battery vitality limit. Wireless sensor organizes additionally have some run of the mill qualities contrasted with these wireless systems. WSN's highlights are appeared as pursues: vitality confinement for hubs utilizing vitality batteries, ability to deal with hub disappointment, hub heterogeneity, adaptability to enormous scale activity, ability to withstand in unfeeling ecological conditions [7].

\subsection{Applications of Wireless Sensor Networks}

Latest wireless framework innovation has made the idea of WSN a reality. It can expand low-power and minimal effort sensor development that can perform different undertakings that connect inside at a small range and are minor in volume. Each center point made out of three things a handset. WSN has been broadly utilized and actualized in different territories in the course of recent years, such as Military purpose, Medical purpose, Home purpose, Habitat purpose and Environmental purpose.

\subsection{Routing Arrangement in Ad-Hoc Networks}

These systems have various steering conventions that can be delegated: cross breed alongside progressive conventions are level directing conventions comprising of on interest just as table-driven steering conventions [8]. The specially appointed system is separated into three gatherings as shown in Figure 2.

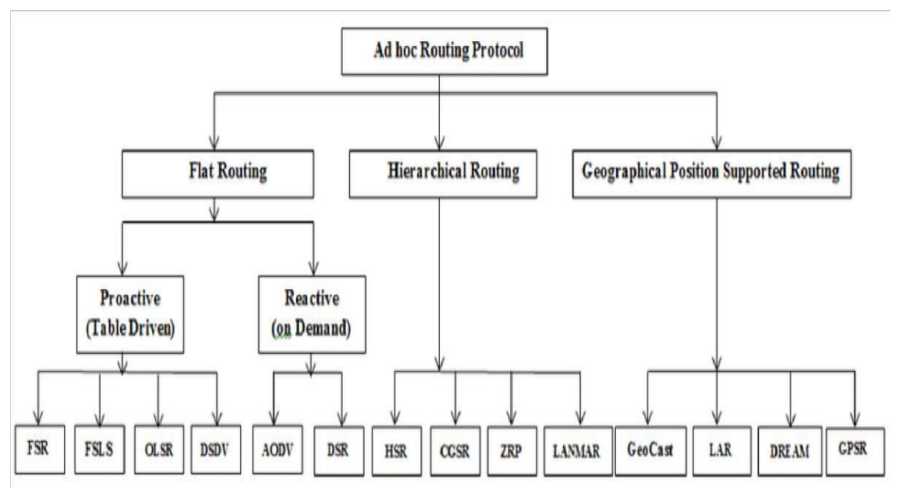

Figure 2: Routing Arrangement 


\section{METHODOLOGY}

In this research, to design the suggested structure, I had adopted WSN's flat network protocols. Using CBR traffic and packet size in WSN for protocol comparison, I used characteristics such as average packet loss, jitter, throughput, information and e2e delay.LEACH needs two tables to be kept up by every hub. These tables are created and kept up by most of the multifaceted nature in LEACH. The updates are occasionally or intended to be transmitted to neighbors as required. As portability increments and the measure of hubs in the system, all the while the size of the transmission capacity and the directing tables expected to refresh these tables increments. Correspondingly, the overhead to keep up and update these tables will improve. Normally, high overhead steering will debase the system's productivity [9].

\section{- Current Status}

The TEEN is a popular routing algorithm scheduled for adhoc networks, but as noted previously, it faces many issues. To date, there has been no commercial implementation for the TEEN, but at UC Berkeley one TEEN simulator is implemented with the $\mathrm{C}++$.

\section{- Advantages}

It is one of the introduced latest simulation algorithm. With fewer nodes, it is suitable to build the adhoc network [10].

\section{- Disadvantages}

The LEACH requires to much of the time update its steering tables utilizing battery vitality alongside a little data transfer capacity regardless of whether the system is latent. Each time the topology of the system changes another succession number is essential before the system is re-shrouded. So LEACH isn't reasonable for profoundly unique systems [11].

\section{A. Performance Matrices}

We have such a large number of quantitative networks that we use to assess different steering convention productivity. In my thesis, I used performance matrices to support the following proactive routing protocols.

\section{B. Throughput}

It is a normal amount of message that is conveyed adequately as a normal amount of bits conveyed every second per unit minute [12].

\section{Average End to End Delay}

It is the extent of the time distinction that cbr parcel sends and gets over the total measure of cbr bundles got to the total time contrast [13].

\section{Latency}

It is depicted as a normal measure of minute between the start of the scattering of an information and the landing of data on the hub that will be keen on getting that data. The time effectiveness for each message is in this way determined by idleness [14].

\subsection{Network Simulators}

These are tools that we use just as recreation for system demonstrating purposes. We have a great deal of test systems that we use to make the circumstance. They help a great deal in estimating system adequacy and other system attributes
[15]. These test systems help organize managers model the system as indicated by their necessities and reenact these models to check the usefulness of the system. System overseers arrange their system for reenactment demonstrating purposes, for example, topology arrangement. System test systems, for example, OMNet++, OPNET, NS-2 and GloMoSim are accessible here. However, after such a large number of long periods of perception and diligent work, I pick ns-2 test system form 2.35. To reproduce the TEEN and LEACH directing conventions, I use ns-2 test system that is flexible and extraordinary for recreation. System test system 2 is the occasion driven system parcel level test system [16], which is being created as a major aspect of the virtual web testbed undertaking created from sun microsystem + UCC Beerkley. Here are two language kinds utilized in ns2, the tcl utilized for frontend just as the $\mathrm{C}++$ utilized for backend.

\subsection{NAM}

NAM is a liveliness instrument dependent on Tcl/TK to view hints of system reenactment and hints of genuine world bundles. A visual understanding of the made system topology is given by NAM. As appeared in Figure 3, it bolsters topology structure, bundle level movement, and diverse data investigation.

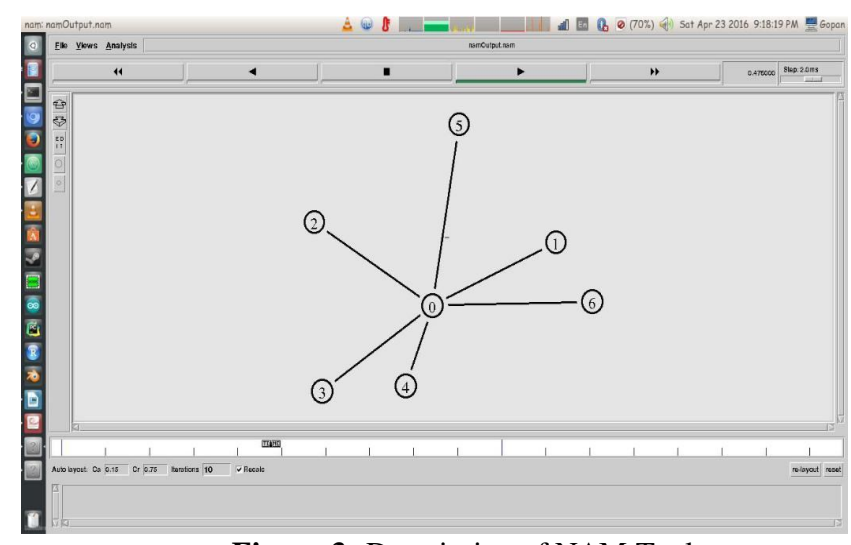

Figure 3: Description of NAM Tool

\subsection{Graphs}

Diagrams are the data plotter for general purposes. There are unmistakable sorts of charts in Microsoft Excel that can likewise deal with the interminable informational collection size and numerous data records as appeared in Figure 4 for planning data for different reports on one diagram. They can share printed yield, store it just as import it into word processor to produce reports and documentation [17]. 


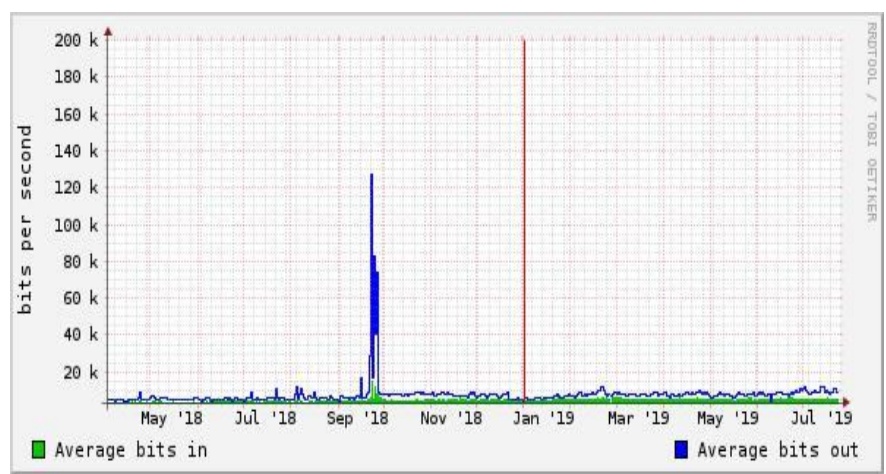

Figure 4: Graphical Representation of data rate.

\subsection{Testing Procedure}

The simulation studies are conducted in the following steps in ns2 for LEACH and TEEN modules:

Ubuntu installation.

Installation plus network simulator settings. Installation plus

NAM setup. Compare the TEEN and LEACH metrics [18].

Make line and bar charts for comparison. I used distinct situations in this experiment and then use the ns 2 to create the study for comparison. In experimental, I took LEACH and TEEN hierarchical routing protocols and made the scenarios dependent on size of the 500,1000, 1500, 2000, 2500, 3000, $3500,4000,4500,5000$ packets to compare the performance, e2e delay, jitter and packet loss of routing protocols using the CBR traffic To display the outcomes, create line charts and bar graphs.

\section{RESULTS}

Throughput of TEEN and LEACH with Packet Size from 500-5000. Table 1 Display the TEEN routing protocol throughput measured according to packet size 500, 1000, 1500, 2000, 2500, 3000, 3500, 4000, 4500, 5000.

Table 1: Throughput TEEN with Packet Size

\begin{tabular}{|l|l|}
\hline Packet Size & TEEN \\
\hline 500 & 233.87 \\
\hline 1000 & 282.98 \\
\hline 1500 & 364.87 \\
\hline 2000 & 341.65 \\
\hline 2500 & 428.4 \\
\hline 3000 & 418.23 \\
\hline 3500 & 373.99 \\
\hline 4000 & 393.83 \\
\hline 4500 & 353.34 \\
\hline
\end{tabular}

\begin{tabular}{|l|l|} 
& \\
\hline 5000 & 317.82 \\
\hline
\end{tabular}

\section{THROUGHPUT TEEN}

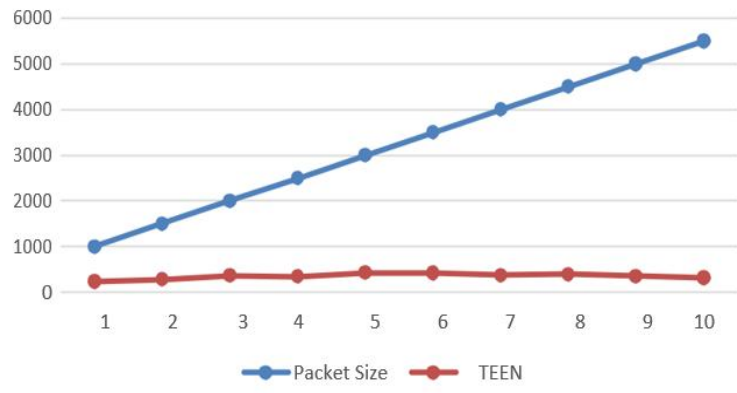

Figure 5: Throughput TEEN with Packet Size

Figure 5 shows the TEEN routing protocol throughput measured according to packet size 500, 1000, 1500, 2000, 2500, 3000, 3500, 4000, 4500, 5000. Throughput is the measure of time required for an information to go through source to destination. In this figure, it can be see that with the increasing of packet size throughput of TEEN is going low and these dots shows the value of TEEN on different packet size.

Table 2: Throughput LEACH with Packet Size

\begin{tabular}{|l|l|}
\hline Packet Size & LEACH \\
\hline 500 & 484.52 \\
\hline 1000 & 386.77 \\
\hline 1500 & 435.26 \\
\hline 2000 & 435.27 \\
\hline 2500 & 768.85 \\
\hline 3000 & 753.25 \\
\hline 3500 & 1146.18 \\
\hline 4000 & 1296.22 \\
\hline 4500 & 1565.5 \\
\hline 5000 & 1639.1 \\
\hline
\end{tabular}


Table 2 Display the LEACH routing protocol throughput measured according to packet size 500, 1000, 1500, 2000, 2500, 3000, 3500, 4000, 4500, 5000

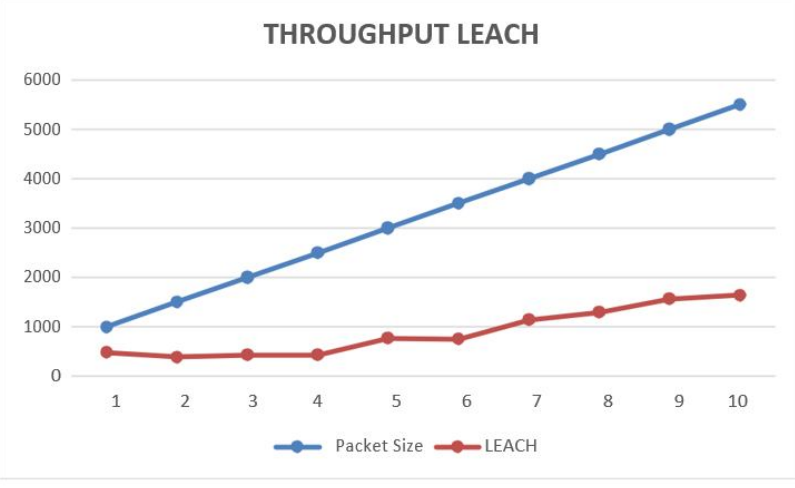

Figure 6: Throughput LEACH with Packet Size Figure 6 shows the LEACH routing protocol throughput measured according to packet size 500, 1000, 1500, 2000, $2500,3000,3500,4000,4500,5000$. Throughput time is the measure of time required for an information to go through an procedure. In this figure, it can be see that with the increasing of packet size throughput of LEACH is going high and these dots shows the value of LEACH on different packet size.

Table 3: Throughput TEEN, LEACH with Packet Size

\begin{tabular}{|c|c|c|}
\hline Packet Size & TEEN & LEACH \\
\hline 500 & 233.87 & 484.52 \\
\hline 1000 & 282.98 & 386.77 \\
\hline 1500 & 364.87 & 435.26 \\
\hline 2000 & 341.65 & 435.27 \\
\hline 2500 & 428.4 & 768.85 \\
\hline 3000 & 418.23 & 753.25 \\
\hline 3500 & 373.99 & 1146.18 \\
\hline 4000 & 393.83 & 1296.22 \\
\hline 4500 & 353.34 & 1565.5 \\
\hline 5000 & 317.82 & 1639.1 \\
\hline
\end{tabular}

Table 3 Displays a graph where the LEACH and TEEN routing protocol differences were assessed with packet sizes of $1000,1500,2000,2500,3000,3500,4000,4500$,

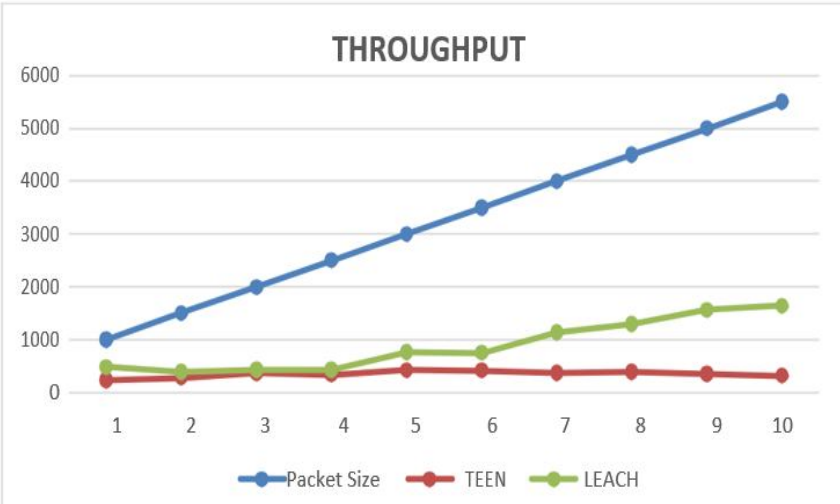

5000.LEACH performance begins to improve at the P.S of 1000 but rising in P.S to 2500 it starts to improve shows that LEACH is higher then TEEN.

Figure 6: Comparison of TEEN, LEACH Throughput with Packet Size

Figure 6 displays a graph where the LEACH and TEEN routing protocol differences were measured with packet sizes of 500, 1000, 1500, 2000, 2500, 3000, 3500, 4000, 4500, 5000. LEACH throughput begins to raise P.S of 1000 but enhance the packet size to 2000 it starts to increase the graph which shows that LEACH throughput is more to TEEN.

\subsection{Jitter of TEEN and LEACH with Packet Size 500 - 5000}

Table 4 Display the TEEN routing protocol jitter measured according to packet size 500, 1000, 1500, 2000, 2500, 3000, 3500, 4000, 4500, 5000 .

Table 4: Jitter TEEN with Packet Size

\begin{tabular}{|l|l|}
\hline Packet Size & TEEN \\
\hline 500 & 248.76 \\
\hline 1000 & 483.56 \\
\hline 1500 & 720.21 \\
\hline 2000 & 793.66 \\
\hline 2500 & 1242.77 \\
\hline 3000 & 1498.24 \\
\hline 3500 & 1625.53 \\
\hline 4000 & 1908.94 \\
\hline 4500 & 2433.78 \\
\hline 5000 & 2764.25 \\
\hline
\end{tabular}

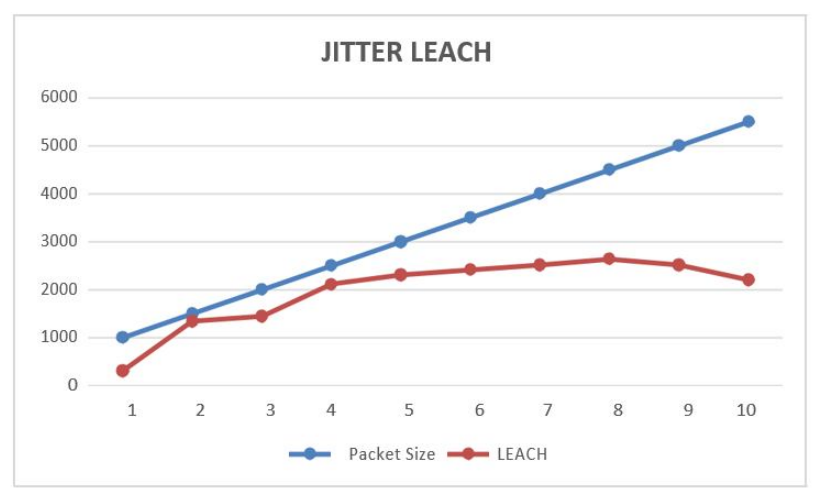

Figure 7: Jitter TEEN with Packet Size

Figure 7: shows the TEEN routing protocol jitter measured according to packet size 500, 1000, 1500, 2000, 2500, 3000, 
$3500,4000,4500,5000$. Jitter in IP systems is the variety in the inactivity on a bundle stream between two frameworks, when a few parcels take more time to venture out from one framework to the next. Jitter results from system blockage, timing float and course changes. In this figure 7 , it can be see that with the increasing of packet size jitter of TEEN is going low and these dots shows the value of TEEN on different packet size.

Table 5: Jitter LEACH with Packet Size

\begin{tabular}{|c|c|}
\hline Table 5: Jitter LEACH with Packet Size \\
\hline Packet Size & LEACH \\
\hline 500 & 307.5 \\
\hline 1000 & 1337.34 \\
\hline 1500 & 1441.32 \\
\hline 2000 & 2110.96 \\
\hline 2500 & 2310.56 \\
\hline 3000 & 2415.03 \\
\hline 3500 & 2510.91 \\
\hline 4000 & 2637.25 \\
\hline 4500 & 2206.62 \\
\hline 5000 & \\
\hline
\end{tabular}

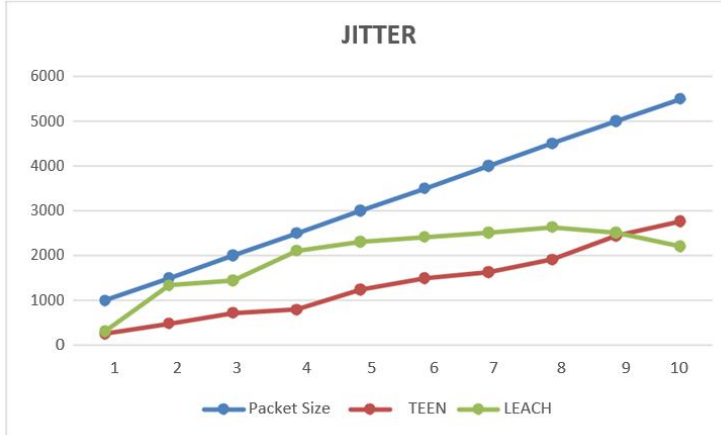

Figure 8: Jitter LEACH with Packet Size

Figure 8 Display the LEACH routing protocol jitter measured according to packet size 500, 1000, 1500, 2000, $2500,3000,3500,4000,4500,5000$. Jitter in IP systems is the variety in the inactivity on a bundle stream between two frameworks, when a few parcels take more time to venture out from one framework to the next. Jitter results from system blockage, timing float and course changes. In this figure 8 , it can be see that with the increasing of packet size jitter of LEACH is going high and these dots shows the value of LEACH on different packet size.
Table 6: Jitter TEEN, LEACH with Packet Size

\begin{tabular}{|c|c|c|}
\hline Packet Size & TEEN & LEACH \\
\hline 500 & 248.76 & 307.5 \\
\hline 1000 & 483.56 & 1337.34 \\
\hline 1500 & 720.21 & 1441.32 \\
\hline 2000 & 793.66 & 2110.96 \\
\hline 2500 & 1242.77 & 2310.56 \\
\hline 3000 & 1498.24 & 2415.03 \\
\hline 3500 & 1625.53 & 2510.91 \\
\hline 4000 & 1908.94 & 2637.25 \\
\hline 4500 & 2433.78 & 2513.42 \\
\hline 5000 & 2764.25 & 2206.62 \\
\hline & & \\
\hline
\end{tabular}

Table 6 Shows TEEN and LEACH routing protocol jitter with packet size. Compared to LEACH, the TEEN took the low jitter rate. The LEACH has a high jitter with a packet size of 1500 , but when the packet size becomes 2000, it begins to increase it at a size of 2500. The LEACH has maximum latency when the P.S risesto 2500 . Then at 4000 it reduces again, but at 4500 it increases. Overall, LEACH will take more jitter to begin the information transfer process.

Figure 9 Shows TEEN and LEACH routing protocol jitter with packet size. Compared to LEACH, the TEEN took the low jitter rate. The LEACH has a high jitter with a packet size of 1500 , but when the packet size becomes 2000, it begins to increase it at a size of 2500 . The LEACH has

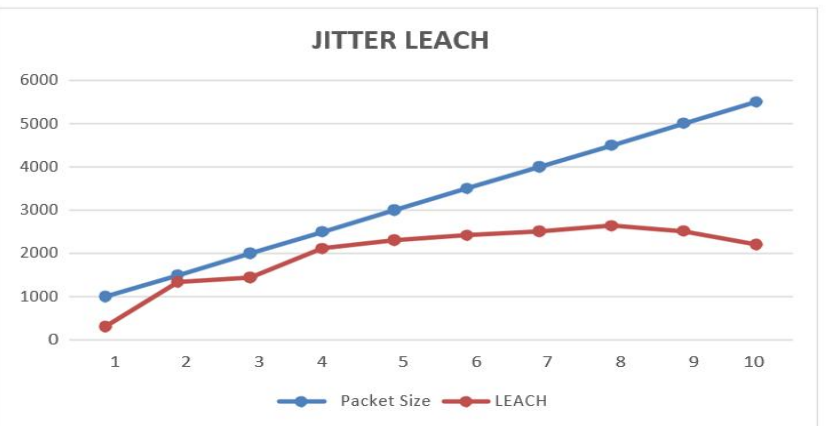

Figure 9: Comparison of TEEN, LEACH Jitter with Packet Size

maximum latency when the packet size rises to 2500 . Then at 4000 it reduces again, but at 4500 it increases. Overall, LEACH will take more jitter to begin the information transfer process.

\subsection{End to End Delay of TEEN and LEACH with Packet Size 500 - 5000}

Table 7 Display the TEEN routing protocol E2E delay measured by packet size 500, 1000, 1500, 2000, 2500, 3000, 3500, 4000, 4500, 5000 . 
Table 7: TEEN End to End Delay with Packet Size

\begin{tabular}{|c|c|}
\hline Packet Size & TEEN \\
\hline 500 & 935.76 \\
\hline 1000 & 1413.7 \\
\hline 1500 & 1453.91 \\
\hline 2000 & 1620.99 \\
\hline 2500 & 1468.53 \\
\hline 3000 & 1470.16 \\
\hline 3500 & 1782.42 \\
\hline 4000 & 2054.68 \\
\hline 4500 & 2157.71 \\
\hline 5000 & 2266.96 \\
\hline
\end{tabular}

Figure 10 Display the TEEN routing protocol E2E delay measured by packet size 500, 1000, 1500, 2000, 2500, 3000, $3500,4000,4500,5000$. It is the normal time taken by an information packet to arrive in the destination. Only the data packets that successfully delivered to destinations that counted. In figure, it can be see that with the increasing of packet size e2e of TEEN is going low and these dots shows the value of TEEN on different packet size.

\subsection{Packet Loss of TEEN and LEACH with Packet Size}

\section{$500-5000$}

Table 8 Display TEEN routing protocol packet loss measured by packet size 500, 1000, 1500, 2000, 2500, 3000, 3500, 4000, 4500, 5000 .

Table 8: Packet Loss of TEEN with Packet Size

\begin{tabular}{|c|c|}
\hline Packet Size & TEEN \\
\hline 500 & 0.07 \\
\hline 1000 & 0.12 \\
\hline 1500 & 0.1 \\
\hline 2000 & 0.11 \\
\hline 2500 & 0.14 \\
\hline 3000 & 0.12 \\
\hline 3500 & 0.2 \\
\hline 4000 & 0.2 \\
\hline 4500 & 0.13 \\
\hline 5000 & 0.12 \\
\hline
\end{tabular}

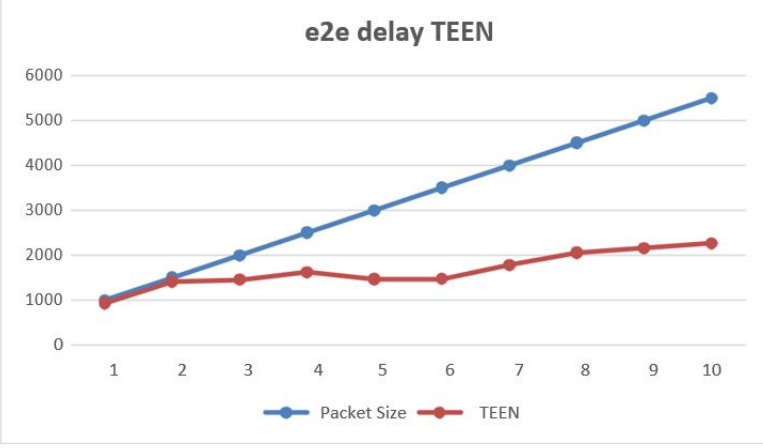

Figure 10: TEEN End to End Delay with Packet Size

Figure 10 Display the TEEN routing protocol E2E delay measured by packet size 500, 1000, 1500, 2000, 2500, 3000, $3500,4000,4500,5000$. It is the normal time taken by an information packet to arrive in the destination. Only the data packets that successfully delivered to destinations that counted. In figure, it can be see that with the increasing of packet size e2e of TEEN is going low and these dots shows the value of TEEN on different packet size.

\subsection{Packet Loss of TEEN and LEACH with Packet Size}

\section{$500-5000$}

Table 8 Display TEEN routing protocol packet loss measured by packet size 500, 1000, 1500, 2000, 2500, 3000, 3500, 4000, 4500, 5000.

Table 8: Packet Loss of TEEN with Packet Size

\begin{tabular}{|c|c|}
\hline Packet Size & TEEN \\
\hline 500 & 0.07 \\
\hline 1000 & 0.12 \\
\hline 1500 & 0.1 \\
\hline 2000 & 0.11 \\
\hline 2500 & 0.14 \\
\hline 3000 & 0.12 \\
\hline 3500 & 0.2 \\
\hline 4000 & 0.2 \\
\hline 4500 & 0.13 \\
\hline 5000 & 0.12 \\
\hline
\end{tabular}


Table 8 Differences in packet loss of TEEN and LEACH for packet sizes $500-5000$, were considered. LEACH packet loss begins to improve at the P.S of 2000 but when we increase the P.S to 2000 it begins to decline to 3000 , which demonstrates that LEACH packet loss is more like TEEN.

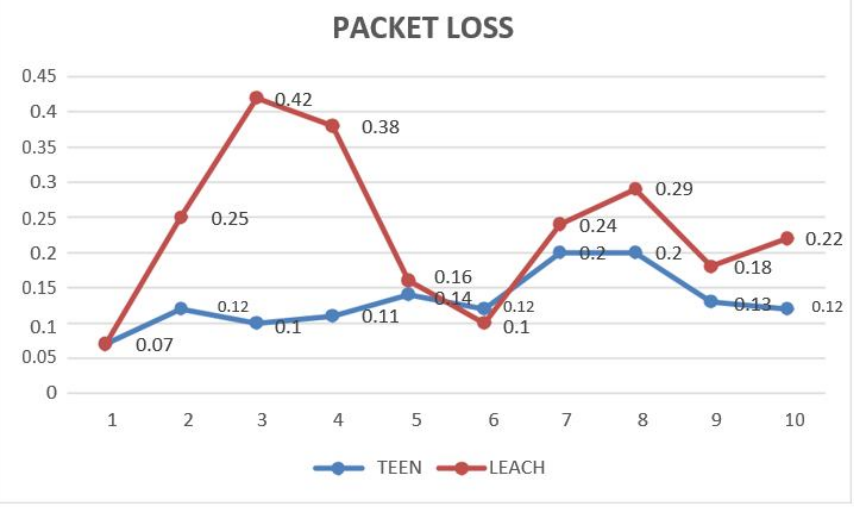

Figure 11: Comparison of Packet Loss of TEEN and LEACH with Packet Size

Figure 11 Differences in packet loss of TEEN and LEACH for packet sizes 500 - 5000, were considered. LEACH packet loss begins to improve at the P.S of 2000 but when we increase the P.S to 2000 it begins to decline to 3000 , which demonstrates that LEACH packet loss is more like TEEN.

\subsection{Comparison of TEEN AND LEACH with Packet Size 500-5000}

Table 9 Displays were considered for packet sizes of 500, 1000, 1500, 2000, 2500, 3000, 3500, 4000, 4500 and 5000 in all parameters of the LEACH and TEEN routing protocol.

Table 9: Comparison of TEEN AND LEACH with Packet Size

\begin{tabular}{|l|l|c|c|c|c|c|c|c|}
\hline Column1 & $\begin{array}{l}\text { Throu } \\
\text { ghput }\end{array}$ & $\begin{array}{c}\text { Colu } \\
\mathrm{mn} 2\end{array}$ & $\begin{array}{c}\text { Colu } \\
\mathrm{mn} 3\end{array}$ & Jitter & $\begin{array}{c}\text { Colu } \\
\mathrm{mn} 4\end{array}$ & $\begin{array}{l}\text { e2e } \\
\text { Delay }\end{array}$ & $\begin{array}{l}\text { Colu } \\
\mathrm{mn} 5\end{array}$ & $\begin{array}{l}\text { Packet } \\
\text { Loss }\end{array}$ \\
\hline Packet Size & T.TEE & $\begin{array}{c}\text { T.LE } \\
\text { ACH }\end{array}$ & $\begin{array}{c}\text { J. } \\
\text { TEE } \\
\mathrm{N}\end{array}$ & $\begin{array}{l}\text { J. } \\
\text { LEA } \\
\text { CH }\end{array}$ & $\begin{array}{c}\text { e2e. } \\
\text { TEE } \\
\mathrm{N}\end{array}$ & $\begin{array}{c}\text { e2e.LE } \\
\text { ACH }\end{array}$ & $\begin{array}{c}\text { P.TE } \\
\text { EN }\end{array}$ & $\begin{array}{c}\text { P.LEA } \\
\text { CH }\end{array}$ \\
\hline 500 & 233.87 & 484.52 & 248.76 & 307.5 & 935.76 & 1094.33 & 0.07 & 0.07 \\
\hline 1000 & 282.98 & 386.77 & 483.56 & 1337.34 & 1413.7 & 2001.02 & 0.12 & 0.25 \\
\hline 2000 & 341.65 & 435.27 & 793.66 & 110.96 & $\begin{array}{c}1620.9 \\
9\end{array}$ & 2260.19 & 0.11 & 0.38 \\
\hline 2500 & 428.4 & 768.85 & 7 & 2310.56 & 1468.5 & 2061.58 & 0.14 & 0.16 \\
\hline 3000 & 418.23 & 753.25 & 1498.2 & 4415.03 & 1470.1 & 1840.59 & 0.12 & 0.1 \\
\hline
\end{tabular}

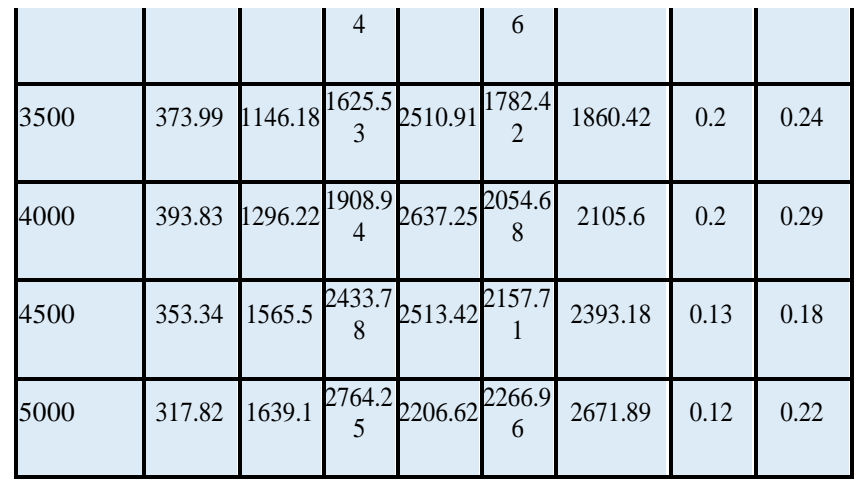

All Parameters

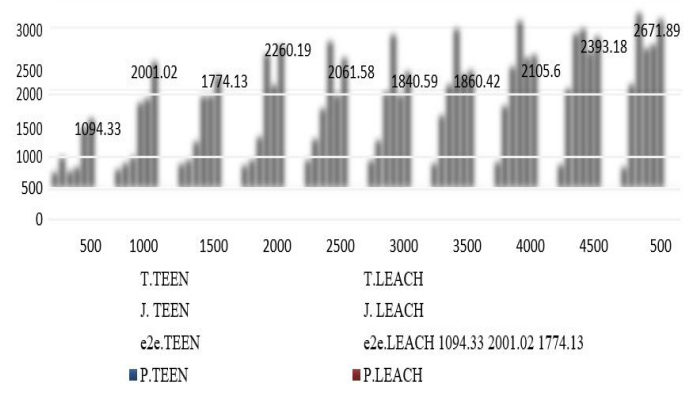

Figure 12: Comparison of TEEN AND LEACH with Packet

Figure 12 shows the differences in all metrics with respect to packet sizes of 500, 1000, 1500 and 2000 in all parameters of the TEEN and $\mathrm{LEACH}$ routing protocol. In figure, it can be see that with the increasing of packet size jitter, e2e delay, throughput and packet loss of LEACH is going high as compare to TEEN and these dots shows the value of TEEN and LEACH on different packet size. As the reenactment discoveries demonstrate that the TEEN throughput is high when compared with LEACH. On top of it, during raising of packet size from $500-5000$, jitter along with e2e delay and packet loss also effected the impact of mobility on protocols. Jitter of LEACH is higher as compared to TEEN along with packet loss. This was examined after performing different experiments based on scenarios. Approximately, we had created ten scenarios file (.tcl) for both protocols which generates the data files (.tr) about jitter, e2e delay and packet loss. On the last, we had concluded that usability of TEEN is better than LEACH on the behalf of different metrics such as jitter, throughput, e2e delay and packet loss.

\section{CONCLUSION}

The wireless sensor network comprise of enormous amounts of little sensor hubs that can procedure, store and transmit data inside a limited range because of their minor size and least value, these hubs with coordinated sensors are usually utilized in different sorts of applications, for example, following and checking of wellbeing. Wireless Sensor Network identifies with a gathering of spatially appropriated and committed sensors to screen and record natural physical conditions and to sort out data accumulated at a key spot. I have analyzed the two steering conventions LEACH and TEEN in wireless sensor arrange based on four measurements throughput, jitter, bundle misfortune and start to finish delay 
with various parcel size of 500, 1000, 1500, 2000, 2500, $3000,3500,4000,4500,5000$. I have made the line diagrams just as the visual charts for every one of the situations first I have made the line diagrams of the considerable number of measurements independently and furthermore made their structured presentations by taking the mean of their qualities and after that we made the examination diagrams of all the I analyzed the two wireless sensor system directing conventions LEACH and TEEN based on four measurements throughput, jitter, parcel misfortune and start to finish delay with various bundle sizes of 500, 1000, 1500, 2000, 2500, $3000,3500,4000,4500,5000$ CBR traffic in NS2.

We made the line diagrams just as the visual diagrams for every one of the circumstances first I delivered the line charts of the considerable number of measurements exclusively and furthermore made their reference charts by taking the mean of their qualities and after that we created the examination diagrams of the considerable number of measurements to comprehend their relative size. In the wake of contrasting LEACH and TEEN conventions, we break down which convention is best directed by interactive media content progressively. These conventions directed different sorts of practices and productivity in different WSN hub versatility rates. Here we evaluate the exhibition concerning the utilization of these normal start to finish deferring measurements, productivity, parcel misfortune and jitter. The results exhibit that the TEEN has raised proficiency, jitter and e2e delay. When contrasted with LEACH in all circumstances, LEACH has e2e delay, low throughput and jitter. TEEN do possess more throughput and alive nodes as the finding of the results. The findings of this study will useful for crucial decision making regarding the selection of the protocol under certain circumstances; such as amount of data, time interval, etc., and for others like military, wireless monitoring or home security and disaster warning.

\section{REFERENCES}

[1] G. F. Ahmed, R. Barskar and N. Barskar. An Improved LEACH Routing Protocol for Wireless Ad Hoc Networks. Procedia Technology, Vol. 6, pp. 822-831, 2012.

[2] K, Akkaya and M. Younis. A survey on routing protocols for wireless sensor networks. Ad Hoc Networks, Vol.3, no.3,pp 325-349, 2005.

[3] L. Alazzawi and A. Elkateeb. Performance Evaluation of the WSN Routing Protocols Scalability. Journal of Computer Systems, Networks and Communications, Vol. 1, no. 1, pp 1-9, 2008.

[4] M. Bhat, S. Swetha and J. T. Devaraju.. A Performance Study of Proactive, Reactive and Hybrid Routing Protocols using Qualnet Simulator. International Journal of Computer Applications, Vol. 28, no. 5, pp.10-17,2011.

[5] M. Z. Hasan, H. Al-Rizzo and M. Gunay. Lifetime maximization by partitioning approach in wireless sensor networks. Eurasip Journal on Wireless Communications and Networking. Vol. 1, no. 1, pp. 1-18, 2017.

[6] Y. K. Hassan, M. H. A. El-Aziz and A. S. A. El-Radi. Performance evaluation of mobility speed over MANET routing protocols, International Journal of Network Security. Vol. 11no. 3, pp. 128-138, 2010.

[7] T. Houngbadji and S. Pierre. QoSNET: An integrated QoS network for routing protocols in large scale wireless sensor networks. Computer Communications, Vol. 33, no. 11, pp. 1334-1342, 2010.

[8] S. Jaggi and E. V. Wasson. Enhanced OLSR Routing Protocol Using Link-Break Prediction Mechanism for WSN. Industrial Engineering \& Management Systems. Vol. 15, no. 3, pp. 259267, 2016.

[9] S. Kalantary and S. Taghipour. A survey on architectures, protocols, applications, and management in wireless sensor networks. Journal of Advanced Computer Science \& Technology, Vol. 3, no. 1, pp. 1-11, 2014.

[10] H. Kaur and Er. J. Singh. Performance comparison of OLSR, GRP and TORA using OPNET. International Journal of Advance Research in Computer and Software Engineering, Vol. 2, no.10, pp. 260-267, 2012.

[11] M. Keshtgary and V. Babaiyan. Performance Evaluation of Reactive, Proactive and Hybrid Routing Protocols. International Journal of Computer Science and Engineering, Vol. 4, no. 2, pp. 248-254, 2012.

[12] D. F. Macedo, A. L. dos Santos, L. H. A. Correia, J. M. Nogueira and G. Pujolle. Transmission power and data rate aware routing on wireless networks. Computer Networks, Vol. 54, no. 17, pp. 2979-2990, 2010.

[13] A. Miran and G. Kadir. Enhancing AODV routing protocol to support QoS, International Journal of Advanced Trends in Computer Science and Engineering (IJATCSE), Vol. 8, no.5, pp. 1824-1830, 2019.

[14] F. J. Blanza and L. Materum. Wireless Propagation Multipath Clustering: On Simultaneously Solving the Membership and the Number of Clusters, International Journal of Advanced Trends in Computer Science and Engineering (IJATCSE), Vol. 8, no.5, pp. 1914-1919, 2019.

[15] M. Ongayev, Z. Sultanova, S. Denizbayev, G. Ozhanov and S. Abisheva. Engineering and Process Infrastructure of the Agro-Industrial Complex, International Journal of Emerging Trends in Engineering Research, Vol. 7, no. 12, pp. 879-885, 2019.

[16] S. Aleksander, A. Konev, T. Kosachenko and D. Dudkin. Threat Model for IoT Systems on the Example of Open UNB Protocol, International Journal of Emerging Trends in Engineering Research, Vol. 7, no. 9, pp. 834-290, 2019.

[17] L. Boggula, B. Navyasri. Energy Efficient Routing Mechanism for Harsh Environment in Wireless Sensor Networks, International Journal of Emerging Trends in Engineering Research, Vol. 7, no. 9, pp. 234-238, 2019.

[18] P. Bakaraniya and S. Mehta, S., 2013. K-LEACH: An improved LEACH protocol for lifetime improvement in WSN. International Journal of Engineering Trends and Technology (IJETT), Vol. 4, no. 5, pp.1521-1526, 2013. 